\title{
Neonatal Fournier's gangrene; sequelly of Traditional birth practice: Case report and Short Review
}

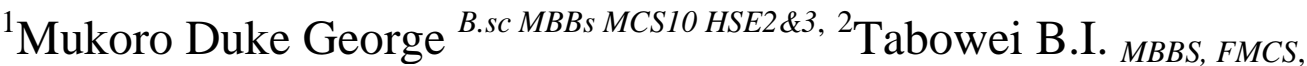 \\ ${ }^{3}$ Olatoregun $\mathrm{F}_{M B B S}$ FRCS, FWACS,FMCS. \\ ${ }^{1,2 \& 3}$ Department of Surgery, Niger-Delta University Teaching Hospital, Okolobiri, Yenogua, Bayelsa ,Nigeria.
}

\begin{abstract}
Fournier's gangrene is one of the infectious and gangrenous diseases seen worldwide, It is commonly reported in Adult males but also in females. Injuries are nidus to its pathogenesis and manymicrobes have been cultured from this clinical entity .This reported case was an incidental presentation resulting from obstetric care given by an unskilled personnel to a high risk pregnancy at term in prolonged labor. The case therefore avails clinician and pediatricians with the opportunity of seeing a rare adult tropical disease of the scrotum in a neonate.

A fourteen day old term male baby presented with multiple perineal lacerations from delivery by a traditional birth attendant to the Surgery Unit. Perineum with scrotum and penis inclusive were noticed to be gangrenous.He was manage by debriment of necrotic tissue, wound dressings, antiseptic solutions (gentian violent, diluted $\mathrm{H}_{2} \mathrm{O}_{2}$ )as well as intravenous antibiotics (ceftriaxone,ciprofloxacin, metronidazole,cloxacillin) ,syrup paracetamol and syrup camoquine, however during the course of management, parent had financial constraints.
\end{abstract}

Fournier's gangrene are rare phenomenon in neonates, and could be a complication that may arise from poor resource countries or communities where traditional birth attendants and their practices strives.

Keywords: Fournier's gangrene, traditional birth attendant, tropical disease, antibiotics, debriment.

\section{Introduction}

In 1764, Baurienne originally described an idiopathic, rapidly progressive soft-tissue necrotizing process that led to gangrene of the male genitalia. However, the disease was named after Jean-Alfred Fournier, a Parisian venereologist, on the basis of a transcript from an 1883 clinical lecture in which Fournier presented a case of perineal gangrene in an otherwise healthy young man, adding this to a compiled series of 4 additional cases $^{1}$.It is generally accepted as synergistic necrotizing fasciitis of perineal, genital, or perianal regions ${ }^{2}$.It has been reported more in males than females ${ }^{2}$. It also occurs in neonates as seen in this case. It was formally thought to be idiopathic ${ }^{3}$, however, studies and reports have proven its pathogenesis to colorectal diseases ${ }^{4}$, urogenital diseases ${ }^{4}$ and Injury from urethal catherization ${ }^{1}$.In rare cases, vasectomy and circumcision and those with Diabetes mellitus ${ }^{2,5}$ have been implicated among the etiologies . Furthermore, Fournier's gangrene could be the presenting sign of an undiagnosed human immunodeficiency virus infection ${ }^{6}$. Darke et $\mathrm{al}^{7}$ in his study of 88 cases stated that anaerobic streptococcus was the organism most frequent culprit. Other organisms that has been cultured are Clostridium ${ }^{7}$ Escherichia coli,Staphylococcal species, Enterobacteriaceae and Fungi. Mixed infection ${ }^{7}$ is usually the case in majority of cases and also in this part of the country. Neonatal forniers gangrene has not been reported, we therefore use this medium to report a rare tropical disease presenting in uncommon age group such as the neonate.

Case Report :A fourteen day old term male baby presented to our health facility with multiple perineal lacerations from attempted delivery by a traditional birth attendant after prolonged labour.

The mother ,a 39 year old grand multipara with obstetric index ;gravida 10 Para 9 , who presented with features of feto-pelvic disproportion, breech presentation and fetal distress at term in prolonged labor from a traditional birth attendant (TBA) place . At the TBA centre, She had series of abdominal massage and several vigorous Vaginal examination and manipulation. She was delivered by emergency ceasarean section after presenting to our Institution(Teaching Hospital). A male baby was delivered with moderate birth asphyxia .Apgar score were 3 and 6 at first and fifth minutes respectively. He was admitted at the special care baby unit in respiratory distress, frog-like position, afebrile ,not pale, anicteric, acyanosed .Anthropometry revealed occipito-frontal circumference of $33.5 \mathrm{~cm}$,supine length of $56 \mathrm{~cm}$ and weight was $3.95 \mathrm{~kg}$.He was conscious but lethargic, anterior fontanelle was flat and normotensive,suck and grasp reflex were absent, reduced tone in lower limbs but normal in upper limbs. He was tachypneic with respiratory rate of 62 cycles per minute. His heart rate was 160 beats per minute. Abdomen was full, umbilical stump clamped clean and dry. Perineum ,penile shaft and prepuce was hyperemic while scrotum was warm to touch ,enlarged ,edematous and transilluminates. 
Seven days later despite wound care and antibiotics, The already tender scrotum started becoming ulcerated with hyper pigmented sloughs, including perineum ${ }^{\text {Figure } 1}$. Baby was resuscitated with fluids ,intranasal oxygen and warmth.Vital signs was monitored with pulse oximeter. His blood count showed leucocytosis $\left(18.4 \times 10^{9} / 1\right)$ with hemoglobin concentration of $15.7 \mathrm{~g} / \mathrm{dl}$. Neutrophil and lymphocyte were 54 and $40 \%$ respectively. Plain radiograph showed no sign of fracture nor dislocation. Surgery Unit were invited,A diagnosis of neonatal forniers gangrene was made. Clinical condition was managed by selective and careful debriment ${ }^{\text {Figure } 2}$ of necrotic tissue ,wound dressings with antiseptic solutions using (gentian violent ,diluted $\mathrm{H}_{2} \mathrm{O}_{2}$ ) as well as intranasal oxygen. Honey was later included only for a short period of time. Other items included in the management were intravenous antibiotics (ceftriaxone, ciprofloxacin, metronidazole,cloxacillin) and intramuscular vitamin $\mathrm{K}$ and tetanus toxiod, syrup paracetamol, and camoquine, also Anti -tetanus serum. However, during the course of management ,parent had financial constraints .Although ,despite the constraint, baby was managed with best minimum care as possible. Scrotum and perineum healed completely with very minimal scar. Testis seemed attached to fibrous tissue within the newly formed scrotal sac .ultrasound scan was requested to follow-up , and relative were also contacted through the phone for follow-up .

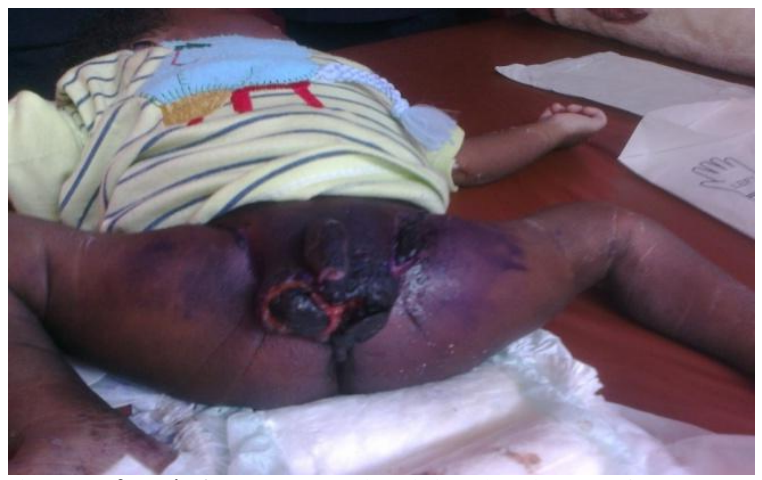

Figure 1: fournier's gangrenous in a injured perineum of a neonate

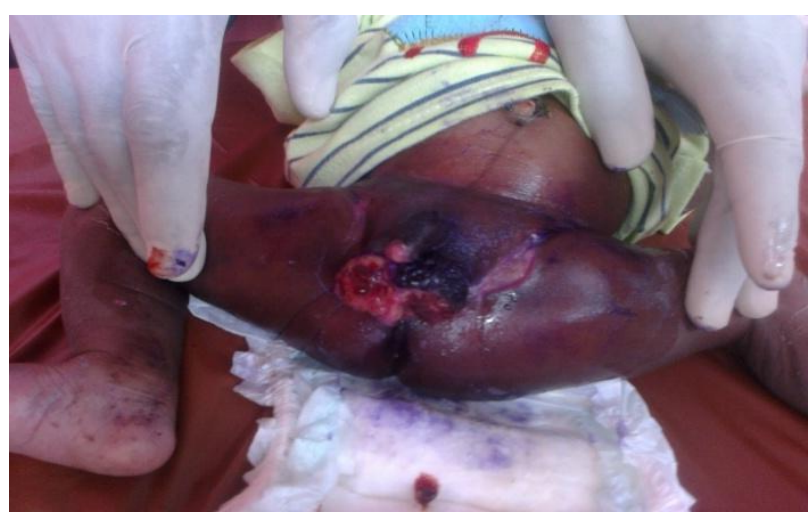

Figure 2:Post debriment of the left cleft and scrotum before dressing

Debriment :patient was cleaned with antiseptic solution and necrotic tissue was selectively excised from the inferior and anterior surface of the scrotum as well as the cleft between the left thigh and perineum . Thereafter wound was dressed and placed in pampers. During most of the days stating from the day of debridement , patient temp swing as high as 37.5 degree and above. However, by the $13^{\text {th }}$ day post-debridement, patient temp had reduced to $37 \mathrm{oC}$. Vital signs were monitored and drugs regimen was charted .

\section{Discussion}

Although originally described as idiopathic gangrene of the genitalia, Fournier gangrene has an identifiable cause in $75-95 \%$ of cases. ${ }^{8}$ The necrotizing process commonly originates from an infection in the anorectum, the urogenital tract, or the skin of the genitalia. ${ }^{9}$ In children, the following have led to the disease: circumcision, strangulated inguinal hernia, Omphalitis, Insect bites, Trauma, Urethral instrumentation, Perirectal abscesses, systemic infections ${ }^{10}$.Neonatal Fournier' gangrene is uncommon .Our patient develop this condition as a result of risk of infection and injury which he was exposed to .Foremost is the prolong rupture of membrane in a traditional attendant(TBAs) place which is usually unsterile for delivery. This condition was considered to be prolong, when considering the delay before presenting to the Teaching Hospital.Secondly ,mother was subjected to aggressive manipulation of the cervix by the traditional birth attendant ,to assist 
delivery possibly with instrument which should be suspected considering the laceration, edema and hyperemia of the scrotum and perineum .These fact can be extracted from the clinical history .There are risk for infection and injury for the fetus before birth .These risk should be suspected when TBAs manipulate breech presentations through the vagina because ,the scrotum and perineum are at risk to injury. Fournier's gangrene is still an important disease with high mortality rates in spite of the developments in intensive care units and newgeneration antibiotics ${ }^{2}$.Although, we were able manage and achieve complete healing in this neonate without skin grafting, this could be as a result of response to antibiotics administered , and debridement to reduced bacterial load , as well as the fast growing tissue of the neonate. Reported treatment modalities which have been established and a available poor resource centres. One of them is debridement ${ }^{7 \& 11}$ which was known to be very effective by Darke and its group in treatment of mixed Clostridia and non-Clostridia infected gangrene .This was the main course of treatment employed in our patient. Furthermore, Darke et al noticed that Hyperbaric oxygen $^{7 \& 11}$ was effective in the treatment of Clostridia infected gangrene. Others have emphasized the use of local honey ${ }^{11}$ application during dressing ${ }^{11}$. Honey is known to have bacteriostatic ${ }^{12 \& 13}$ and bacteriocidal ${ }^{12 \& 13}$ effect as well as osmotic effect thereby removing excess fluid and improving microcirculation .Honey has been shown to be effective against Gram-positive bacteria (Staphylococcus aureus, Bacillus subtilis, Bacillus cereus, Enterococcus faecalis, and Micrococcus luteus) ${ }^{12}$ and Gram-negative bacteria (Escherichia coli, Pseudomonas aeruginosa, and Salmonella typhi $)^{12}$. One more emphasis is the use of adequate prophylaxis with penicillin ${ }^{7}$ in patients at risk as stated by Darke. This may be due to anaerobic streptococcus that has been implicated, however on clinical ground it may not be easily distinguished from clostridium infected gangrene.

Conclusively, Fournier's gangrene in neonate is one of the sequelly that may follow injury to scrotum and perineum in attending to breech delivery by unskilled personnel .

\section{References:}

[1]. Corman JM. Classic articles in colonic and rectal surgery. Dis Colon Rectum. 1988:31:984-8

[2]. Atil Ç, Volkan G, Cihangir A, Ayhan K H and Selçuk M. H; Fournier's gangrene: Is it scrotal gangrene? Advances in Therapy, Volume 25, Number 10 (2008), 1065-1074, DOI: 10.1007/s12325-008-0103-1

[3]. López PJ C, Sánchez M JM, Piñeiro F MC, Bouso M M, Parra M L, García A J ;Fournier's gangrene secondary to urethral catheterization. Arch Esp Urol. 2005 Mar;58(2):167-70.

[4]. Basoglu M, Ozbey I, Atamanalp SS, Yildirgan MI, Aydinli B, Polat O, Ozturk G, Peker K, Onbas O, Oren D. Management of Fournier's gangrene: review of 45 cases. Surg Today. 2007;37(7):558-63. Epub 2007 Jun 26.

[5]. Lamerton A.J. : Fournier's gangrene: non-clostridial gas gangrene of the perineum and diabetes mellitus.J R Soc Med. 1986 April; 79(4): 212-215. PMCID: PMC1290277

[6]. McKay TC, Waters WB: Fournier's gangrene as the presenting sign of an undiagnosed human immunodeficiency virus infection. J Urol. 1994 Nov;152(5 Pt 1):1552-4. PMID:7933199

[7]. Darke SG, King AM, Slack WK :Gas gangrene and related infection; classification, clinical features and aetiology, management and mortality. A report of 88 cases.Br J Surg. 1977 Feb;64(2):104-12

[8]. Clayton MD, Fowler JE Jr, Sharifi R, Pearl RK. Causes, presentation and survival of fifty-seven patients with

[9]. Gamagami RA, Mostafavi M, Gamagami A, Lazorthes F. Fournier's gangrene: an unusual presentation for rectal carcinoma. Am J Gastroenterol. Apr 1998;93(4):657-8. [Medline].

[10]. Vernon M Pais Jr, Bradley F. S., Thomas Santora, Daniel B Rukstalis: Andrew A Aronson,Alex Jacocks, Richard Lavely,Eric L Legome, Michael T Marynowski, David L Morris,Erik D Schraga, Richard H Sinert, Francisco Talavera, Todd Thomsen: Fournier Gangrene ,Medscape reference,Drug ,Disease,Procedure,up-dated,8 Dec 2011.

[11]. N. Eke, Fournier's gangrene: a review of 1726 cases British Journal of Surgery, Volume 87, Issue 6, pages 718-728, June 2000

[12]. D. P. Mohapatra, ${ }^{1}$ V. Thakur, ${ }^{2}$ and S. K. Brar ${ }^{1, *}$; Antibacterial Efficacy of Raw and Processed Honey :Biotechnol Res Int. 2011; 2011: 917505. Published online 2010 December 29. doi: 10.4061/2011/917505

[13]. Nicholas Namias: Honey in the Management of Infections ; Mary Ann Liebert inc : Surgical infections; Volume 4, number 2, 2003. 